\title{
ENDOR-Induced EPR of Disordered Systems: Application to X-Irradiated Alanine
}

\author{
Jevgenij Kusakovskij, Kwinten Maes, Freddy Callens, Henk Vrielinck* \\ Ghent University, Department of Solid State Sciences, Krijgslaan 281-S1, 9000 Ghent, Belgium \\ E-mail: Henk.Vrielinck@ugent.be \\ Phone: +3292644356
}

\begin{abstract}
The electron paramagnetic resonance (EPR) spectra of radiation-induced radicals in organic solids are generally composed of multiple components that largely overlap due to their similar weak $g$ anisotropy and a large number of hyperfine (HF) interactions. Such properties make these systems difficult to study using standard cw EPR spectroscopy even in single crystals. Electron-nuclear double resonance (ENDOR) spectroscopy is a powerful and widely used complementary technique. In particular, ENDOR-induced EPR (EIE) experiments are useful for separating the overlapping contributions. In the present work, these techniques were employed to study the EPR spectrum of stable radicals in X-irradiated alanine, which is widely used in dosimetric applications. The principal values of all major proton HF interactions of the dominant radicals were determined by analyzing the magnetic field dependence of the ENDOR spectrum at $50 \mathrm{~K}$, where the rotation of methyl groups is frozen. Accurate simulations of the EPR spectrum were performed after the major components were separated using an EIE analysis. As a result, new evidence in favor of the model of the second dominant radical was obtained.
\end{abstract}

\section{INTRODUCTION}

L- $\alpha$-alanine (Figure $1 \mathrm{~A}$ ) is the second most common non-essential amino acid in the human body. ${ }^{1}$ Under biological conditions, it is made up of three chemical groups - a protonated amino, a deprotonated carboxyl and a methyl group - bound to a $\mathrm{C}$ atom, labeled as $\mathrm{C}_{\alpha}$. The first reports of the radiation-induced electron paramagnetic resonance (EPR) spectrum of this amino acid date from the early sixties, ${ }^{2,3}$ when alanine and other amino acids were employed to gain insight into the nature of hyperfine (HF) interactions in organic molecules. It was almost immediately realized that solid-state L- $\alpha$-alanine is particularly interesting for dosimetric applications ${ }^{4}$ and today it is fully recognized as a secondary radiation dose standard. ${ }^{5-8}$ In view of this, considerable effort has been devoted to the understanding of the radiation chemistry of alanine and to reliable simulations of its powder EPR spectrum at room temperature (RT).

The EPR spectrum of stable radiation-induced radicals in alanine crystals was convincingly decomposed by Sagstuen and co-workers in an electron-nuclear double resonance (ENDOR) and ENDOR-induced EPR (EIE) study, primarily focused on the proton HF interactions. ${ }^{9}$ The authors have demonstrated that the EPR spectrum can be explained using contributions of three distinct radical species, two of which are dominant. These radicals were labeled as R1-R3 and their chemical structures can be found in Figure 1 B-D, respectively. Decomposition of the dosimetric powder spectrum was accomplished by exploiting the different decay rates of the three species at elevated temperatures ${ }^{10,11}$ and simulations of the complete spectrum became possible only after the $\tilde{g}$ tensors of the stable radicals had been determined. ${ }^{12}$

The ${ }^{14} \mathrm{~N}$ HF interactions are smaller than the majority of the ${ }^{1} \mathrm{H}$ interactions and they are often neglected in simulations of the powder EPR spectrum at RT, when reproduction of the main features of the spectrum is the main aim. ${ }^{9}$ Inclusion of these interactions can, nevertheless, be necessary in quantitative applications, where even the fine details of the spectrum shape need to be reproduced. In addition, the structural information that these interactions encode provides additional evidence for the radical models. For these

\footnotetext{
*To whom correspondence should be addressed
} 
reasons, the ${ }^{14} \mathrm{~N}$ interactions of R1 and R2 were experimentally determined by Rakvin and Maltar-Strmečki using pulsed EPR spectroscopy. ${ }^{13,14}$

Density functional theory (DFT) calculations and related methods, alongside general radiation chemistry principles, have and are still employed to unravel the radiation chemistry of the radicals in irradiated alanine. First computational studies of these radicals were primarily concerned with determining their geometries on isolated molecules. ${ }^{15,16}$ Reaction pathways leading to the formation of R1, R2 and R3 from primary species were devised afterwards by Sagstuen and co-workers ${ }^{17}$ using general radiation chemistry principles. Radical R1 is one of the best studied radicals in the field of radiation chemistry and it was tentatively identified as a deamination product soon after the stable EPR spectrum was recorded for the first time. ${ }^{18-21}$ The model of R1 was confirmed using periodic DFT calculations after most of its spin Hamiltonian parameters were accurately determined ${ }^{22}$ and its reaction chain was later corrected using nudged elastic band simulations. ${ }^{23}$ In Ref. [9], radical R2 and the minority species R3 were convincingly identified as an $\mathrm{H}_{\alpha}$-abstraction radical and a $\mathrm{NH}_{2} \mathrm{C}^{\bullet}\left(\mathrm{CH}_{3}\right) \mathrm{COO}(\mathrm{H})$ radical (Figure 1), respectively, but they were not studied as extensively as the deamination product $\mathrm{R} 1$ in recent years.

It is interesting to note that the experimental parameters of the EPR spectrum of irradiated alanine at cryogenic temperatures were examined less meticulously than the RT ones. This knowledge can, however, be very helpful in computational studies like the one in Ref. [23]. The proton HF interaction tensors of the frozen methyl group of R1 are known from single crystal EPR ${ }^{21}$ and computational ${ }^{22}$ studies, which have some inherent uncertainties associated with them. The non-averaged methyl couplings of R2 and R3 have not been experimentally determined, but the principal values of these interaction tensors were calculated by a number of researchers using $a b$ initio and semi-empirical methods. ${ }^{15,24-26}$ Finally, the reference frames of the methyl interactions of R1 and R2, required for correct averaging of these couplings, have not been reported until now.

Here, we present the results of our efforts to complete the knowledge of the two dominant stable alanine radicals and to simultaneously assess the possibilities of ENDOR spectroscopy for decomposing EPR spectra in disordered systems. The latter was accomplished by analyzing the magnetic field dependence of the cw ENDOR spectrum, also known as the field-frequency ENDOR (FFENDOR) spectrum. The final output of this analysis was then compared to the results of previous studies. The reference frames of methyl interactions of R1 and R2 were obtained using a validated DFT calculation methodology. ${ }^{22,23,27}$ Sufficiently accurate simulations of the EPR spectrum at cryogenic temperatures were made after the spin Hamiltonian parameters of R1 and R2 were refined in an EIE-like analysis suited for disordered systems. Finally, new experimental and computational evidence in favor of the model of R2 were obtained.

\section{Experimental ANd Computational Details}

An irradiated L- $\alpha$-alanine (or simply alanine) sample was taken from a set prepared by Vanhaelewyn et al. for a statistical decomposition and thermal annealing study. ${ }^{10}$ The powders of analytically pure alanine were irradiated at RT with X-rays, produced by a $10 \mathrm{MeV}$ electron beam impinging on a tantalum-graphite target. The sample exposed to a $70 \mathrm{kGy}$ dose was used here because it offered a good compromise between a sufficiently intense EPR spectrum and an almost negligible line broadening. The former is desirable for higher intensity of the ENDOR response. The latter, on the other hand, is known to negatively affect the resolution of the EPR spectrum and might also be an indication of increased disorder or decreased spin lattice relaxation time, which can lead to difficulties with saturating the EPR transitions. For more details about the sample, the reader is referred to Ref. [10].

The EPR and ENDOR spectra were recorded at $50 \mathrm{~K}$ in Q-band ( $34 \mathrm{GHz})$ on a Bruker ElexSys E500 spectrometer equipped with a Pendulum CNT-90XL frequency counter and an Oxford CF935 He-flow cryostat (2-300 K). Magnetic fields were measured with a Bruker ER 035 NMR gaussmeter and calibrated against the $g_{\perp}=2.0031$ component of a $\mathrm{CO}_{3}^{3-}$ radical in irradiated calcite $\left(\mathrm{CaCO}_{3}\right){ }^{28}$ The temperature of $50 \mathrm{~K}$ was chosen to freeze out the rotation of the methyl group, which complicates the interpretation of ENDOR and EIE spectra via its influence on the relaxation properties of the radiation induced radicals in

irradiated alanine. ${ }^{9}$ Other experimental parameters were then determined by optimization. EPR spectra 
were recorded at $2 \mu \mathrm{W}$ power, $12.5 \mathrm{kHz}$ modulation frequency and $0.1 \mathrm{mT}$ modulation amplitude. ENDOR spectra were recorded at $0.2 \mathrm{~mW}$ microwave power, $2.5 \mathrm{~W}$ RF power and $250 \mathrm{MHz}$ modulation depth.

Fits and simulations of spectra were performed with MATLAB ${ }^{\circledR}$ routines using second order perturbation theory, as implemented in EasySpin. ${ }^{29}$ Decomposition of overlapping components in the FFENDOR spectrum was performed by independently fitting a simplified Hamiltonian

$$
\hat{H}=\mu_{B} g_{e} B_{0} \hat{S}_{z}+A_{x} \hat{S}_{x} \hat{I}_{x}+A_{x} \hat{S}_{y} \hat{I}_{y}+A_{x} \hat{S}_{z} \hat{I}_{z}-g_{N} \mu_{N} B_{0} \hat{I}_{z}
$$

an isotropic ENDOR linewidth and an amplitude for every component contributing to the ENDOR spectrum at every magnetic field. This amounted to 5 parameters per component for a given magnetic field value. Lorentzian functions were fitted to artifact lines, which occurred at a limited set of field-independent frequency positions. The fitted lines were then subtracted from the experimental spectra for presentation. The starting values of all fitting parameters were visually approximated at every magnetic field and the optimizations were carried out using the Levenberg-Marquardt algorithm. ${ }^{30,31}$

For the DFT calculations of all spin Hamiltonian parameters, the crystal structure of L-alanine was taken from the neutron diffraction study by Lehmann et al. ${ }^{32}$ The crystals are known to be orthorhombic with space group $P 2{ }_{1} 2_{1} 2_{1}$, which implies four molecules in a unit cell with lattice parameters: $a=0.6025 \mathrm{~nm}$, $b=1.2324 \mathrm{~nm}$ and $c=0.5783 \mathrm{~nm}$. The $\langle a b c>$ orthogonal reference frame was chosen in accordance with previous experimental and theoretical work of other researchers. ${ }^{9,23}$ DFT calculations were performed using the periodic approach as implemented in the $\mathrm{CP} 2 \mathrm{~K}$ software package. $\mathrm{A}<2 a b 2 c>$ supercell, BLYP functional and a 350 Ry plane wave cut-off energy were used, by following the methodology of Pauwels and co-workers. ${ }^{22,23}$ The scaling approximation after Van Yperen-De Deyne et al. ${ }^{33}$ was employed for the calculation of $\tilde{g}$ tensors, in accordance with our work on radicals in irradiated sucrose. ${ }^{27,34}$

\section{REsUlts}

The Q-band EPR spectrum of X-irradiated alanine powder is shown in Figure 2, where it can be seen that the entire spectrum fits in a narrow magnetic field range (ca. $12.5 \mathrm{mT}$ ). This implies weak yet non-negligible $g$ anisotropy and dominance of HF interactions of all major spectral components, which is a typical characteristic of C-centered radicals. Even without prior knowledge of the radical composition, the multicomponent nature of the spectrum can be anticipated from a considerable number of subtle deviations from a perfectly symmetric shape. Inferring the exact number of components from the EPR spectrum alone would, however, be difficult due to the equally subtle effects of the $g$ anisotropy. All things considered, this spectrum can be seen as a representative example of a radiation-induced spectrum of a generic organic solid to be studied with ENDOR spectroscopy.

\section{III.A. Magnetic Field Dependence of the ENDOR Spectrum}

The FFENDOR spectrum of proton interactions of radicals in X-irradiated alanine is presented in Figure 3. At least $9 \mathrm{HF}$ interactions with small anisotropy can be readily distinguished, i.e. those corresponding to patterns $7-8 \mathrm{MHz}$ wide on the RF axis. This observation immediately reveals that strong interactions with $\beta$-hydroxyl protons, whose anisotropy typically spans the range of ca. $12 \mathrm{MHz}$, do not contribute to the spectrum. It can also be seen that the widths on the magnetic field axis of the 9 distinguished patterns noticeably differ, confirming the supposition that several distinct species contribute to the spectrum. Visual comparison of the patterns reveals that there are two contributions: one that is $11.4 \mathrm{mT}$ wide and a noticeably broader one of $12.5 \mathrm{mT}$. This suggests that contributions of one of the known radicals, most likely the ones of the minority species R3, were not detected due to the lower sensitivity of ENDOR spectroscopy. The observed lines are now going to be labeled and assigned.

Radical R1. Three of the aforementioned patterns can be assigned to the first species. Centers of these spectral components are found at 59, 90 and $111 \mathrm{MHz}$, which corresponds to HF interactions of 15, 77 and $120 \mathrm{MHz}$. These interactions will be labeled as R1 $\beta 3, \mathrm{R} 1 \beta 2$ and $\mathrm{R} 1 \beta 1$, respectively. Their weak anisotropy and the fact that they average to $71 \mathrm{MHz}$ suggest that these protons are bound to $\mathrm{C}_{\beta}$ atoms. ${ }^{35}$ Their sum is 
$212 \mathrm{MHz}$ (or $7.6 \mathrm{mT}$ ), which is much less than the total width of the spectrum in the magnetic field domain. This inconsistency implies that at least one major HF interaction is missing in the initial assessment. Closer inspection of the FFENDOR spectrum (Figure 3) reveals a broad feature spanning the 65-96 MHz range that is $11.4 \mathrm{mT}$ wide, which corresponds to a HF interaction with $A_{\min }=27 \mathrm{MHz}$ and $A_{\max }=89 \mathrm{MHz}$. This interaction can be identified as an $\alpha$ coupling $(\mathrm{R} 1 \alpha)$ by the $|58| \mathrm{MHz}$ isotropic part if we assume a typical $\alpha$ anisotropy, i.e. $(a-b, a, a+b)$. Simultaneous presence of an $\alpha$ and $3 \beta$ couplings enable the identification of this species as the deamination product (R1).

Radical R2. The remaining 6 weakly anisotropic components can all be assigned to the second species. The patterns centered around 57, 58, 66, 86, 95 and $117 \mathrm{MHz}$ correspond to 11, 13, 30, 69, 88 and $131 \mathrm{MHz}$ $\beta$-proton interactions, respectively. The sum of these interactions is $342 \mathrm{MHz}$ (or $12.2 \mathrm{mT}$ ) and it accounts for almost the complete width of the corresponding spectral component. The average of these is $57 \mathrm{MHz}$, which is significantly smaller than the $70-80 \mathrm{MHz}$ expected for $\mathrm{C}_{\beta}$-bound protons. The chemical structure of the molecule, on the other hand, suggests that $\mathrm{N}_{\beta}$-bound nuclei can also be expected and it is known that the proton interactions of the $\mathrm{N}_{\beta} \mathrm{H}_{3}^{+}$group average to $50-60 \mathrm{MHz}$. ${ }^{35}$ With this in mind, these interactions should be divided into two groups that are associated with $-\mathrm{C}_{\beta} \mathrm{H}_{3}$ and $-\mathrm{N}_{\beta} \mathrm{H}_{3}^{+}$fragments. With the previous findings, the species can now be identified as a radical formed by an $\mathrm{H}$ atom abstraction from the $\mathrm{C}_{\alpha}$ atom (R2).

At this point, the grouping of the 6 interactions assigned to R2 is ambiguous if one solely relies on the isotropic parts of HF interactions. This can be resolved if the RF domain widths of these components, related to the interaction anisotropy, are examined. In a number of combined experimental and computational studies of different amino acids, ${ }^{35-38}$ the anisotropy of the amino proton couplings appears to be systematically larger than the anisotropy of the methyl couplings. It can be seen in Figure 3 that the patterns positioned around 57, 66 and $95 \mathrm{MHz}$ are slightly broader than the ones around 58, 86 and $117 \mathrm{MHz}$. Thus, the former three can be assigned to the amino group and labeled as R2a $33, \mathrm{R} 2 \mathrm{a} \beta 2$ and R2a $\beta 1$, respectively, and the latter - to the methyl group and labeled as $\mathrm{R} 2 \mathrm{~m} \beta 3, \mathrm{R} 2 \mathrm{~m} \beta 2$ and $\mathrm{R} 2 \mathrm{~m} \beta 1$, respectively. To our knowledge, this property has not been formally proven and could be a feature unique to amino acids. To be sure that the assignments of HF interactions for both radicals were correct, they have to be verified by confronting the experimental data with DFT calculation results. This can be done after the principal values of the HF interactions are known with higher precision.

Principal values of HF tensors. To exploit the high resolution of ENDOR spectroscopy, sets of Hamiltonians like the one in Eq. 1 were fitted to the patterns assigned to the $9 \beta$-proton interactions in the FFENDOR spectrum (Figure 3). In this way, the principal values of interaction tensors $\left(A_{x}<A_{y}<A_{z}\right)$ were determined for all magnetic fields. By taking the smallest realizations of $A_{x}$ and the largest ones of $A_{y}$ and $A_{z}$, more accurate estimates were obtained. Uncertainties of these parameters can be estimated from selected slices of the FFENDOR spectrum with the highest amplitudes with the usual methods. In the present case, it is sufficient to estimate only the upper bound of uncertainties on all principal values $\Delta A_{i} \approx 0.1 \mathrm{MHz}$.

The fitting results are summarized in Tables 1 and 2 together with own DFT calculation results. In these tables, own results are also compared to experimental and computational findings from previous investigations. The agreement is good, so it can be concluded that all resolved patterns in the FFENDOR spectrum (Figure 3) have now been identified and that no features associated with the R3 radical were observed.

\section{III.B. ENDOR-Induced EPR}

After the principal values of all major HF interactions have been determined, the EPR spectrum of the system under study can be simulated. By keeping these parameters fixed, the principal values of the $\tilde{g}$ tensors can be estimated, which generally provides further constraints on the radical models and presents a consistency check of data that were already gathered.

Suppose, for example, that the interaction with the $\alpha-{ }^{1} \mathrm{H}$ nucleus of $\mathrm{R} 1$ was missed during the analysis of the field dependence of the ENDOR spectrum. This would result in poor agreement of the simulated and experimental EPR spectra, as can be seen from spectra A and D in Figure 4. In principle, it is possible 
to resolve this discrepancy by solely relying on simulations of the EPR spectrum. The analysis can be simplified, however, if contributions of the two radicals were separated. This can be accomplished with a slightly unconventional use of EIE that we have introduced in our study of the fourth stable radical in irradiated sucrose. ${ }^{39}$

The higher frequency range of the RF domain of the FFENDOR spectrum in Figure 3 was fitted using 5 components with Hamiltonians as in Eq. 1 for the radical contributions. The experimental spectra and the fitted surfaces, presented as black contour plots, are shown in Figure 3B. Out of the 5 surfaces obtained in this way, 2 are associated with R1 and the 3 remaining ones - with R2. These surfaces were individually integrated over the RF domain twice, which produced absorption-like traces typical to EIE experiments. To reduce the noise, the obtained EIE spectra were averaged for the respective components. The resulting EIE spectra are presented in Figure 4 as traces E and I for the radicals R1 and R2, respectively. The contributions of the two radicals can now be analyzed independently.

The entire set of HF interactions from Tables 1 and 2 was employed in the simulations depicted in Figure 4 as traces C, G and K. These spectra were obtained after manually tweaking the relative radical contributions, isotropic line widths and principal values of the $\tilde{g}$ tensors. The most satisfying agreement was achieved with $(2.0036,2.0031,2.0020) \pm 0.0003$ and $(2.0038,2.0032,2.0022) \pm 0.0004$ principal $g$ values, and 3:2 relative contributions for R1 and R2, respectively. It should be noted that this 3:2 ratio of R1:R2 EPR spectral components does not (necessarily) reflect the ratio of the concentrations for the two radical species, in view of the differences in $T_{1}$ and $T_{2}$ relaxation times for the radicals. The alignment of the principal directions of the R1 $\alpha$ interaction relatively to the corresponding $\tilde{g}$ tensor was chosen with the two-center dipole approximation in mind. ${ }^{35}$ The overall agreement of simulations with the experiment is remarkably good, which can be attributed to the weak anisotropy of the majority of the spin Hamiltonian parameters. Inclusion of the ${ }^{14} \mathrm{~N}$ HF tensors from Refs. [13] and [14] in the simulations did not lead to noticeable improvements in the agreement with the experiment. Inspection of the EIE spectra and corresponding simulations reveals that the discrepancies are largest for the R1 component, which exhibits the anisotropic $\alpha$-interaction.

\section{Discussion}

The species selectivity of the ENDOR effect was exploited to decompose a rather complicated spectrum of an irradiated alanine powder, dominated by overlapping contributions of radicals R1 and R2. The minority species R3 was not detected here, which not surprisingly shows that the sensitivity of a powder analysis is generally lower than the one of a single-crystal study. It has to be noted, however, that the aforementioned radical was difficult to characterize even in single crystals due to the weakness of its relative contribution $(<10 \%)$ and the simultaneous presence of several conformations. ${ }^{9}$ Its spectrum was only recently isolated in a statistical decomposition study of the EPR spectrum. ${ }^{11}$ In more fortunate cases, e.g. when the EPR components of minority species are narrow and easier to distinguish, better sensitivity could be expected.

Decomposition of the magnetic field dependence of the ENDOR spectrum using simplified Hamiltonians enabled the separation of the contributions of the 5 largest HF interactions, 2 of which were ascribed to $\mathrm{R} 1$ and the remaining $3-$ to R2. Double integration of these components over the RF domain produced EIE-like spectra of the two contributing species. Using the obtained EIE spectra and the HF interactions determined in the perturbation theory analysis of the FFENDOR spectrum, it was possible to determine the principal values of the $\tilde{g}$ tensors and to simulate the EPR spectrum sufficiently well. The determined relative contributions of R1 and R2 and the principal values of the $\tilde{g}$ tensor of R2 agree perfectly with the Q-band results reported in Ref.. ${ }^{12}$ The $g$ values of R1, on the other hand, were slightly underestimated in our work. These discrepancies probably stem from the large anisotropy of the $\alpha$-interaction and the lower accuracy of the powder analysis.

An attempt was made to determine the relative orientations of the tensor frames of R1 $\beta 1$ and $R 2 \mathrm{~m} \beta 1 \mathrm{HF}$ interactions with respect to the corresponding $\tilde{g}$ tensors to see if these parameters can be extracted from the 
experimental FFENDOR data. A more general spin Hamiltonian

$$
\hat{H}=\mu_{B} \hat{\vec{S}} \cdot \tilde{g} \cdot \vec{B}+\sum_{j=1}^{N}\left(\hat{\vec{I}}_{j} \cdot \tilde{A}_{j} \cdot \hat{\vec{S}}-g_{N} \mu_{N} \hat{\overrightarrow{I_{j}}} \cdot \vec{B}\right)
$$

was fitted to the 108-122 MHz segment of the spectrum by varying the principal values and the tilting angles of the two relevant HF tensors. The parameters of all other significant HF interactions and $\tilde{g}$ tensors were kept fixed to the values determined in the FFENDOR and EIE analysis, and coinciding frames were assumed. The starting values of the tilting angles were chosen to be close to the DFT-predicted ones to increase the chances of success of this endeavor. The output of the fitting routine was then compared to the results of DFT calculations. The final outcome of the procedure outlined above was, however, inconclusive. Large deviation angles between a considerable number of fitted principal directions from the respective DFT predictions were observed and it was impossible to find systematic reasons for these discrepancies.

It seems that at least two factors have to be investigated more thoroughly before decisive conclusions can be made about the feasibility of determining the directional information of interaction tensors with the neighboring nuclei in the disordered state. These factors are the ill-posedness of the fitting problem and the fact that the neglect of tilts of the smaller HF tensors in the simulations of FFENDOR spectra can strongly influence the outcome of a fitting routine. The latter problem can in principle be explained by the fact that the orientation selection depends on all terms in the Hamiltonian to a varying degree. If several unknown terms are not treated carefully, incorrect orientations are selected at different magnetic fields during simulations of ENDOR spectra. This problem can potentially be avoided by varying more parameters of the spin Hamiltonian, while fitting the EPR (or the EIE) spectrum together with the FFENDOR spectra of as many dominant HF interactions assigned to this species as possible. At this point in time, this procedure is too computationally expensive, but the situation can change in the foreseeable future after more efficient simulation algorithms are developed.

Even though it was not possible to experimentally determine the principal directions of the HF interactions, it is still interesting to illustrate how they influence the EPR spectrum in the present case. Fortunately, the principal directions of the HF interactions are known to be reproduced very accurately with DFT for radicals in amino acids. ${ }^{22,23,37,38}$ The predictions of the principal values of these tensors, on the other hand, are known to have less absolute precision. It is thus worth performing the simulations of the EPR powder spectrum from before, but with the DFT-predicted tensor eigenframes. Simulations of the EPR spectrum using the relative tensor orientations predicted by DFT calculations (Supplementary Information) are shown in Figure 4 as traces B, F and J. The visual agreement of the simulated spectra of R1 and R2 with the corresponding EIE spectra (Figure $4 \mathrm{E}$ and I) has slightly improved, but there is hardly any improvement in the case of the complete EPR spectrum (Figure $4 \mathrm{~A}$ ). This shows that fitting these parameters using the EPR spectrum alone would be practically impossible, and that even with separated contributions and field dependence of ENDOR spectra it would be far from trivial.

In this work, the principal values of all significant proton HF interactions of R1 and R2 were determined with high accuracy by analyzing the FFENDOR spectrum. Good agreement of the obtained data with the previous experimental and computational studies can be reported (Tables 1 and 2). The principal values of $3 \tilde{A}$ tensors assigned to the frozen methyl group of $\mathrm{R} 2$ presented here have not been reported before and serve as additional evidence in favor of the radical model, which so far has not been validated as rigorously as the one of R1. ${ }^{23}$

Further evidence for the model of R2 can be found in Table 3, where the calculated HF interaction tensor of the ${ }^{14} \mathrm{~N}$ nucleus and the average $\mathrm{HF}$ tensor of the methyl protons $\left({ }^{1} \mathrm{H}_{\beta}\right.$-av. $)$ are compared to the appropriate experimental results of other researchers. Overall, the agreement is very good. The isotropic part of the ${ }^{14} \mathrm{~N}$ interaction is only slightly underestimated if the sign ambiguity was not resolved in the experimental data. The calculated anisotropy of the principal values and the principal directions of this interaction, on the other hand, are in excellent agreement with the experiment. Same is true for all of the parameters of the ${ }^{1} \mathrm{H}_{\beta}$-av. tensor, which makes the case for the current radical model of $\mathrm{R} 2$ even stronger. This knowledge will potentially be useful in computational studies of radical transformations as in Ref. [23], 
where the structures of observable (semi-)stable species are crucial for inferring the intermediate radical states.

To conclude this discussion, it should be noted that the data set obtained here is substantial and that it would have been sufficient for devising radical models if they were not known. This is quite remarkable and encouraging for investigations of radiation-induced radicals in other systems, such as DNA in the solid state, where single crystals are not available. In general and especially when dealing with macro biomolecules, one might not be so fortunate to achieve unambiguous identifications and additional constraints would be required. The obtained data would still be of use while devising further experiments.

\section{Conclusions}

In summary, EPR and ENDOR spectroscopies were employed to study the radiation-induced radicals in powder alanine. Analysis of the magnetic field dependence of the ENDOR spectrum provided accurate values of all dominant proton HF interactions of the two dominant radical species. Generalized EIE experiments enabled the partial decomposition of the EPR spectrum, which in turn led to improved simulations of the complete spectrum. The method of separation of overlapping spectral components presented here can be readily applied to other disordered systems of organic radicals and it is not limited to cw ENDOR spectroscopy. DFT calculations were employed to validate the results of the analysis and to obtain reliable estimates of the tensor frames of all significant HF interactions. The latter are available in the Supplementary Information. In addition, new evidence in favor of the model of the second dominant radical species was obtained. Computational studies of the chemical reaction path of this radical are likely to benefit from this knowledge.

\section{ACKNOWLEDGMENTS}

Authors are grateful to Professor Stefan Stoll for the support with the EasySpin simulation software, to Professor Einar Sagstuen for providing insight into the alanine literature and to Professor Sabine Van Doorslaer for the discussions on the ${ }^{14} \mathrm{~N}$ interaction of the $\mathrm{R} 2$ radical. The computational resources (STEVIN Supercomputer Infrastructure) and services used in this work were kindly provided by Ghent University, the Flemish Supercomputer Center (VSC), the Hercules Foundation and the Flemish Government - department EWI. JK gratefully acknowledges the financial support of the Ghent University's Special Research Fund (BOF).

\section{Supporting Information Available}

Calculated $\tilde{g}$ and HF interactions of radicals R1 and R2.

\section{REFERENCES}

(1) Doolittle, R. F. In Prediction of Protein Structure and the Principles of Protein Conformation, Fasman, G. D., Ed.; Springer US: 1989, pp 599-623.

(2) Heller, C.; McConnell, H. M. Radiation Damage in Organic Crystals. II. Electron Spin Resonance of $\left(\mathrm{CO}_{2} \mathrm{H}\right) \mathrm{CH}_{2} \mathrm{CH}\left(\mathrm{CO}_{2} \mathrm{H}\right)$ in $\beta$-Succinic Acid. J. Chem. Phys. 1960, 32, 1535-1539.

(3) Horsfield, A.; Morton, J. R.; Whiffen, D. H. Identification of the Radical $\bullet \mathrm{CH}_{2}\left(\mathrm{CO}_{2} \mathrm{H}\right)$. Nature 1961, 189, 481-481.

(4) Bradshaw, W. W.; Cadena, D. G.; Crawford, G. W.; Spetzler, H. A. W. The Use of Alanine as a Solid Dosimeter. Radiat. Res. 1962, 17, 11-21.

(5) Nam, J. W.; Regulla, D. F. The Significance of the International Dose Assurance Service for Radiation Processing. Int. J. Rad. Appl. Instrum. A 1989, 40, 953-955. 
(6) Anton, M.; Allisy-Roberts, P. J.; Kessler, C.; Burns, D. T. A Blind Test of the Alanine Dosimetry Secondary Standard of the PTB Conducted by the BIPM. Metrologia 2014, 51, 06001.

(7) Desrosiers, M. F. In Applications of EPR in Radiation Research, Lund, A., Shiotani, M., Eds.; Springer International Publishing: 2014, pp 489-507.

(8) Goodman, B. A.; Worasith, N.; Ninlaphruk, S.; Mungpayaban, H.; Deng, W. Radiation Dosimetry Using Alanine and Electron Paramagnetic Resonance (EPR) Spectroscopy: A New Look at an Old Topic. Appl. Magn. Reson. 2016, 1-19.

(9) Sagstuen, E.; Hole, E. O.; Haugedal, S. R.; Nelson, W. H. Alanine Radicals: Structure Determination by EPR and ENDOR of Single Crystals X-Irradiated at 295 K. J. Phys. Chem. A 1997, 101, 9763-9772.

(10) Vanhaelewyn, G.; Sadlo, J.; Callens, F.; Mondelaers, W.; De Frenne, D.; Matthys, P. A Decomposition Study of the EPR Spectrum of Irradiated Sucrose. Appl. Radiat. Isotopes 2000, 52, 1221-1227.

(11) Jåstad, E. O.; Torheim, T.; Villeneuve, K. M.; Kvaal, K.; Hole, E. O.; Sagstuen, E.; Malinen, E.; Futsaether, C. M. In Quest of the Alanine R3 Radical: Multivariate EPR Spectral Analyses of X-Irradiated Alanine in the Solid State. J. Phys. Chem. A 2017, 121, 7139-7147.

(12) Heydari, M. Z.; Malinen, E.; Hole, E. O.; Sagstuen, E. Alanine Radicals. 2. The Composite Polycrystalline Alanine EPR Spectrum Studied by ENDOR, Thermal Annealing, and Spectrum Simulations. J. Phys. Chem. A 2002, 106, 8971-8977.

(13) Rakvin, B.; Maltar-Strmečki, N. Study of the First Stable L-Alanine Paramagnetic Center by 2DHYSCORE Spectroscopy: Detection of ${ }^{14} \mathrm{~N}$ Hyperfine and Quadrupole Splitting. Chem. Phys. Lett. 2005, 415, 161-164.

(14) Maltar-Strmečki, N.; Rakvin, B. Investigation of the Nitrogen Hyperfine Coupling of the Second Stable Radical in $\gamma$-Irradiated L-Alanine Crystals by 2D-HYSCORE Spectroscopy. J. Magn. Reson. 2012, 222, 81-87.

(15) Lahorte, P.; De Proft, F.; Vanhaelewyn, G.; Masschaele, B.; Cauwels, P.; Callens, F.; Geerlings, P.; Mondelaers, W. Density Functional Calculations of Hyperfine Coupling Constants in Alanine-Derived Radicals. J. Phys. Chem. A 1999, 103, 6650-6657.

(16) Ban, F.; Wetmore, S. D.; Boyd, R. J. A Density-Functional Theory Investigation of the Radiation Products of L- $\alpha$-Alanine. J. Phys. Chem. A 1999, 103, 4303-4308.

(17) Sagstuen, E.; Sanderud, A.; Hole, E. O. The Solid-State Radiation Chemistry of Simple Amino Acids, Revisited. Radiat. Res. 2004, 162, 112-119.

(18) Miyagawa, I.; Gordy, W. Electron Spin Resonance of an Irradiated Single Crystal of Alanine: SecondOrder Effects in Free Radical Resonances. J. Chem. Phys. 1960, 32, 255-263.

(19) Horsfield, A.; Morton, J. R.; Whiffen, D. H. The Electron Spin Resonance Spectrum of $\mathrm{CH}_{3} \mathrm{C}^{\bullet} \mathrm{HCOOH}$ at $77 \mathrm{~K}$ in L- $\alpha$-Alanine. Molecular Physics 1961, 4, 425-431.

(20) Morton, J. R.; Horsfield, A. Electron Spin Resonance Spectrum and Structure of $\mathrm{CH}_{3} \mathrm{C} \bullet \mathrm{H}\left(\mathrm{CO}_{2} \mathrm{H}\right)$. The Journal of Chemical Physics 1961, 35, 1142-1143.

(21) Miyagawa, I.; Itoh, K. Electron Spin Resonance of Irradiated Single Crystals of Alanines: Hindered Rotation of the Methyl Group in a Free Radical. J. Chem. Phys. 1962, 36, 2157-2163.

(22) Pauwels, E.; De Cooman, H.; Waroquier, M.; Hole, E. O.; Sagstuen, E. On the identity of the radiationinduced stable alanine radical. Phys. Chem. Chem. Phys. 2010, 12, 8733-8736.

(23) Pauwels, E.; De Cooman, H.; Waroquier, M.; Hole, E. O.; Sagstuen, E. Solved? The Reductive Radiation Chemistry of Alanine. Phys. Chem. Chem. Phys. 2014, 16, 2475-2482.

(24) Pauwels, E.; Van Speybroeck, V.; Lahorte, P.; Waroquier, M. Density Functional Calculations on AlanineDerived Radicals: Influence of Molecular Environment on EPR Hyperfine Coupling Constants. J. Phys. Chem. A 2001, 105, 8794-8804. 
(25) Petrenko, T. L. Transformation and Structure of Cation Radicals in L- $\alpha$-Alanine. J. Phys. Chem. A 2002, 106, 149-156.

(26) Simion, C. Theoretical Investigation of Radical Species Formed from L- $\alpha$-Alanine Under GammaIrradiation. J. Radioanal. Nucl. Chem. 2008, 275, 331-335.

(27) Kusakovskij, J.; De Cooman, H.; Sagstuen, E.; Callens, F.; Vrielinck, H. On the Identity of the Last Known Stable Radical in X-Irradiated Sucrose. Chem. Phys. Lett. 2017, 674, 6-10.

(28) Serway, R. A.; Marshall, S. A. Electron Spin Resonance Absorption Spectra of $\mathrm{CO}_{3}{ }^{-}$and $\mathrm{CO}_{3}{ }^{3-}$ Molecule-Ions in Irradiated Single-Crystal Calcite. J. Chem. Phys. 1967, 46, 1949-1952.

(29) Stoll, S.; Schweiger, A. EasySpin, a Comprehensive Software Package for Spectral Simulation and Analysis in EPR. J. Magn. Reson. 2006, 178, 42-55.

(30) Marquardt, D. An Algorithm for Least-Squares Estimation of Nonlinear Parameters. J. Soc. Ind. Appl. Math. 1963, 11, 431-441.

(31) Press, W. H.; Teukolsky, S. A.; Vetterling, W. T.; Flannery, B. P., Numerical Recipes: The Art of Scientific Computing, 3rd; Cambridge University Press: Cambridge, UK; New York, 2007.

(32) Lehmann, M. S.; Koetzle, T. F.; Hamilton, W. C. Precision Neutron Diffraction Structure Determination of Protein and Nucleic Acid Components. I. Crystal and Molecular Structure of the Amino Acid L-Alanine. J. Am. Chem. Soc. 1972, 94, 2657-2660.

(33) Van Yperen-De Deyne, A.; Pauwels, E.; Van Speybroeck, V.; Waroquier, M. Accurate Spin-Orbit and Spin-Other-Orbit Contributions to the g-Tensor for Transition Metal Containing Systems. Phys. Chem. Chem. Phys. 2012, 14, 10690-10704.

(34) Kusakovskij, J.; Callens, F.; Vrielinck, H. EMR Study and DFT-Assisted Identification of Transient Radicals in X-Irradiated Crystalline Sucrose. J. Phys. Chem. B 2015, 119, 6562-6570.

(35) Gordy, W., Theory and Applications of Electron Spin Resonance; John Wiley \& Sons Inc: New York, 1980; $638 \mathrm{pp}$.

(36) Vanhaelewyn, G.; Vrielinck, H.; Callens, F. Room Temperature Q-Band Electron Magnetic Resonance Study of Radicals in X-Ray-Irradiated L-Threonine Single Crystals. Radiat. Prot. Dosim. 2014, 159, 155-163.

(37) Øyen, L. F.; Aalbergsjø, S. G.; Knudtsen, I. S.; Hole, E. O.; Sagstuen, E. Direct Radiation Effects to the Amino Acid Side Chain: EMR and Periodic DFT of X-Irradiated 1-Asparagine at 6 K. J. Phys. Chem. B 2015, 119, 491-502.

(38) Knudtsen, I. S.; Aalbergsjø, S. G.; Hole, E. O.; Sagstuen, E. Radiation Effects on Crystalline LAsparagine, Revisited: Radical Formation by EMR and Periodic DFT after X-Irradiation at $275 \mathrm{~K}$. Radiat. Phys. Chem. 2015, 106, 151-159.

(39) Kusakovskij, J.; Caretti, I.; Van Doorslaer, S.; Callens, F.; Vrielinck, H. Fourth Stable Radical Species in X-Irradiated Solid-State Sucrose. Phys. Chem. Chem. Phys. 2016, 18, 10983-10991. 


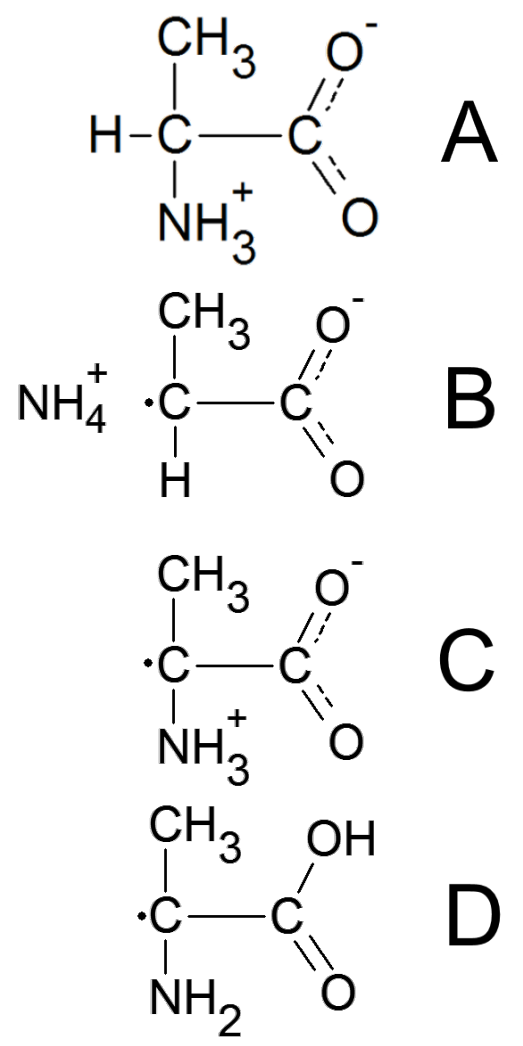

Figure 1: Chemical structure of (A) an alanine molecule in the solid state and the stable radicals formed after irradiation - (B) R1, (C) R2 and (D) R3. 


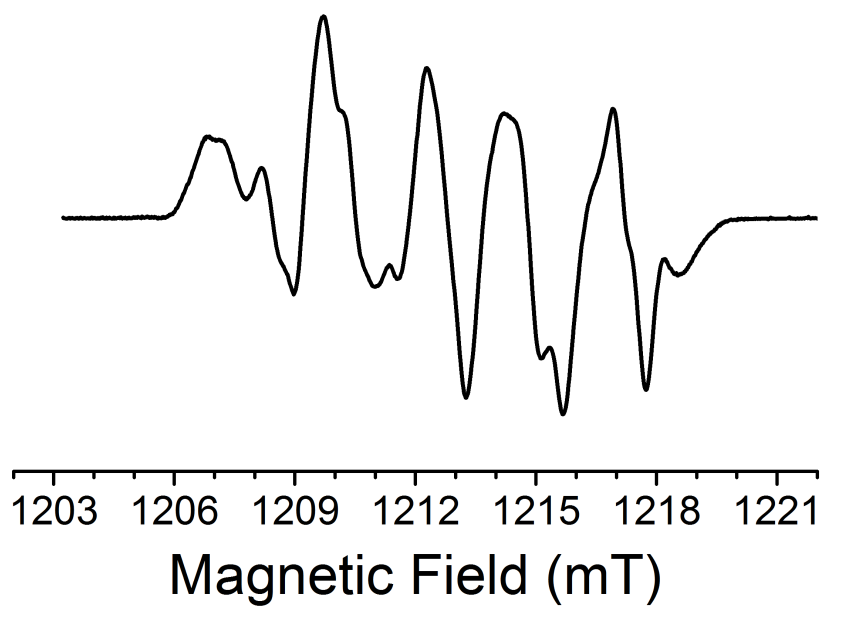

Figure 2: The Q-band EPR powder spectrum of X-irradiated alanine at $50 \mathrm{~K}$. 


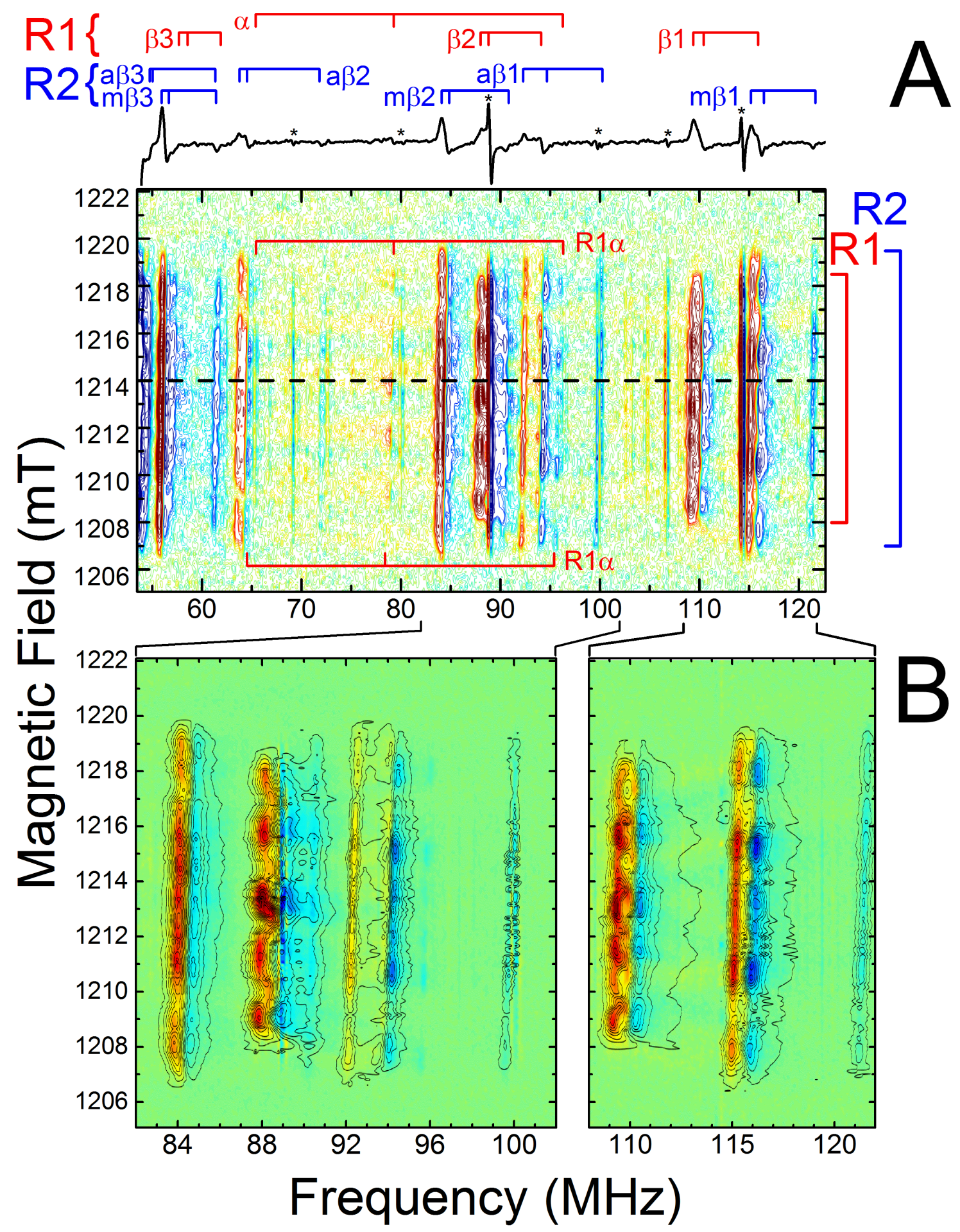

Figure 3: (A) Contributions of 10 components can be identified in the FFENDOR spectrum of X-irradiated alanine, presented here as a contour plot. The components can be assigned to 2 radical species, as is shown at the top of the figure. For the purpose of illustrating how complex individual ENDOR spectra can be, an ENDOR spectrum recorded at $1214 \mathrm{mT}$ is shown using a black line. Field independent artifact lines are labeled with asterisks $\left(^{*}\right)$. (B) The 82-102 and the 108-122 MHz ranges, shown here as color plots, were decomposed into components by independently fitting the Hamiltonian in Eq. 1 for all magnetic fields. The fitted surfaces are presented as black contour lines on top of the experimental data and it can be seen that the agreement is very good. 


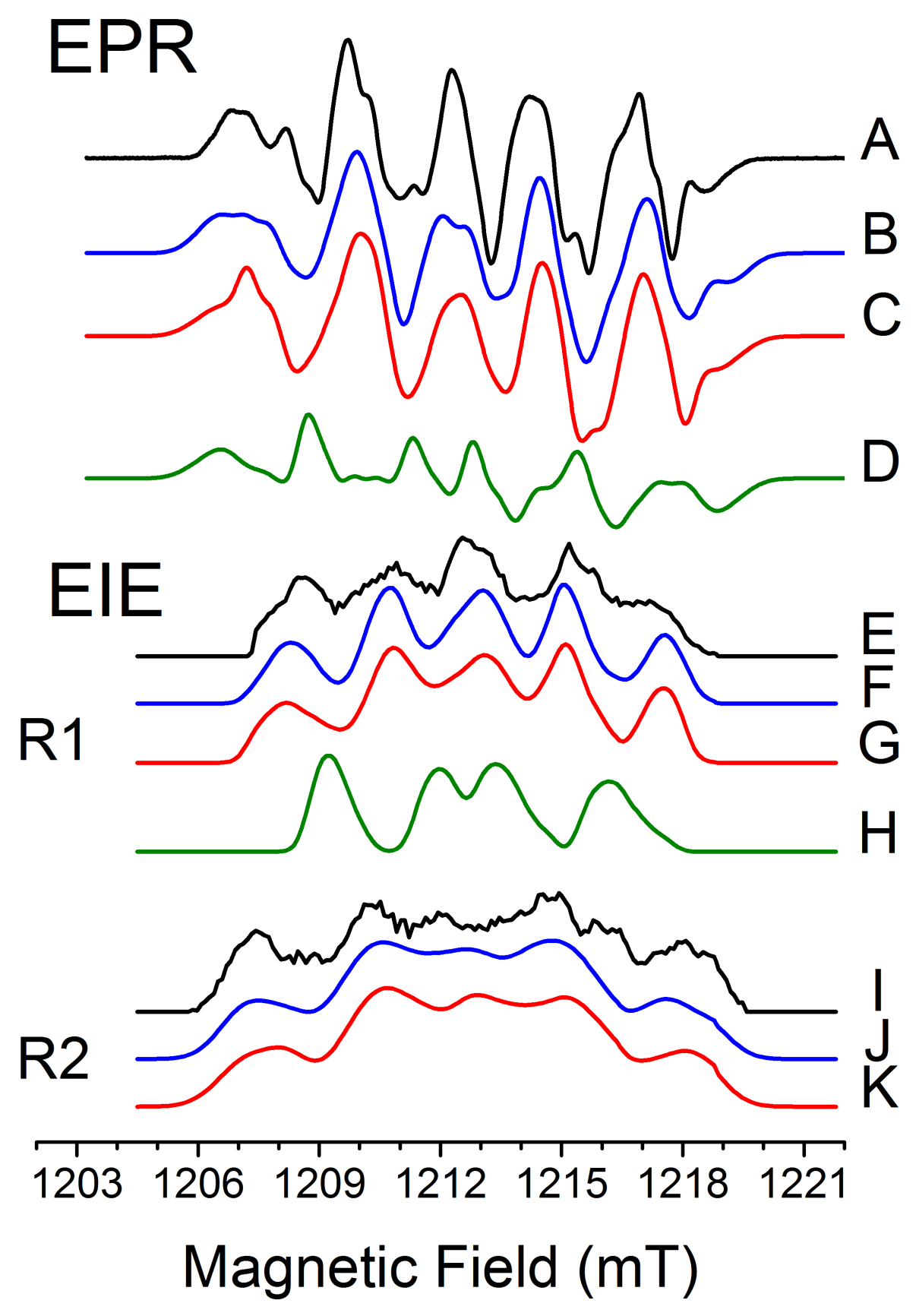

Figure 4: The Q-band EPR powder spectrum of X-irradiated alanine (A) is dominated by two components. Integrated EIE spectra of these components, obtained in a decomposition of the FFENDOR spectra (Figure 3), can be assigned to R1 (E) and R2 (I). All simulations were performed using HF interactions from Tables 1 and 2. Line widths of $0.6 \mathrm{mT}$ and $1.0 \mathrm{mT},(2.0036,2.0031,2.0020)$ and $(2.0038,2.0032,2.0022) \tilde{g}$ tensors, and relative contributions of 3:2 were assumed for R1 and R2, respectively. Simulations presented with blue lines took into account the relative orientations of all tensors by using DFT predictions (B, F, J) and the ones presented with red lines assumed that all tensors have coinciding frames $(\mathrm{C}, \mathrm{G}, \mathrm{K})$. The spectra displayed using green lines illustrate how the spectrum would have changed if the $\alpha$ coupling of R1 was missed in the analysis of the FFENDOR spectrum $(D, H)$. 
Table 1: Comparison of principal values (expressed in MHz) of the HF interactions of the R1 radical in irradiated alanine. Complete tensors obtained with DFT calculations are available in Table S1 in the Supplementary Information.

\begin{tabular}{|c|c|c|c|c|c|c|c|c|c|c|}
\hline \multirow{2}{*}{ Tensor } & \multicolumn{2}{|c|}{ Own Exp. } & \multicolumn{2}{|c|}{ Own DFT } & \multirow{2}{*}{$\begin{array}{l}\text { Ref. [9] } \\
A_{\text {iso }}\end{array}$} & (ENDOR) & \multicolumn{2}{|c|}{ Ref. [23] (DFT) } & \multicolumn{2}{|c|}{ Ref. [21] (EPR) } \\
\hline & $A_{\text {iso }}$ & $A_{\text {aniso }}$ & $A_{\text {iso }}$ & $A_{\text {aniso }}$ & & $A_{\text {aniso }}$ & $A_{\text {iso }}$ & $A_{\text {aniso }}$ & $A_{\text {iso }}$ & $A_{\text {aniso }}$ \\
\hline \multirow[t]{3}{*}{$\mathrm{R} 1 \alpha$} & & 2.2 & & -2.9 & & 3.9 & & -2.5 & & \\
\hline & -56.9 & -31.7 & -46.0 & -30.3 & -56.1 & -31.8 & -42.3 & -30.0 & & \\
\hline & & 29.5 & & 33.2 & & 27.9 & & 32.6 & & \\
\hline \multirow[t]{3}{*}{$\mathrm{R} 1 \beta 1$} & & -4.8 & & -5.4 & & & & -5.3 & & -4.7 \\
\hline & 120.0 & -3.1 & 121.1 & -2.8 & & & 118.8 & -2.8 & 121.3 & -3.3 \\
\hline & & 7.9 & & 8.2 & & & & 8.0 & & 7.9 \\
\hline \multirow[t]{3}{*}{$\mathrm{R} 1 \beta 2$} & & -4.7 & & -4.9 & & & & -5.0 & & -3.9 \\
\hline & 77.3 & -3.4 & 80.9 & -2.8 & & & 93.5 & -2.7 & 77.6 & -2.5 \\
\hline & & 8.1 & & 7.7 & & & & 7.8 & & 6.5 \\
\hline \multirow[t]{3}{*}{$\mathrm{R} 1 \beta 3$} & & -4.7 & & -3.8 & & & & -3.6 & & -4.7 \\
\hline & 14.8 & -2.0 & 5.6 & -2.9 & & & 2.6 & -3.0 & 14.8 & 0.3 \\
\hline & & 6.7 & & 6.7 & & & & 6.7 & & 4.5 \\
\hline
\end{tabular}


Table 2: Comparison of principal values (expressed in $\mathrm{MHz}$ ) of the HF interactions of the R2 radical in irradiated alanine. Complete tensors obtained with DFT calculations are available in Table S2 in the Supplementary Information.

\begin{tabular}{|c|c|c|c|c|c|c|c|c|}
\hline \multirow{2}{*}{ Tensor } & \multicolumn{2}{|c|}{ Own Exp. } & \multicolumn{2}{|c|}{ Own DFT } & \multirow{2}{*}{$\begin{array}{l}\text { Ref. [9] } \\
A_{\text {iso }}\end{array}$} & \multirow{2}{*}{$\begin{array}{c}\text { (ENDOR) } \\
A_{\text {aniso }}\end{array}$} & \multicolumn{2}{|c|}{ Ref. [15] (DFT) } \\
\hline & $A_{\text {iso }}$ & $A_{\text {aniso }}$ & $A_{\text {iso }}$ & $A_{\text {aniso }}$ & & & $A_{\text {iso }}$ & $A_{\text {aniso }}$ \\
\hline \multirow[t]{3}{*}{$\mathrm{R} 2 \mathrm{a} \beta 1$} & & -6.3 & & -6.1 & & -6.8 & & -5.5 \\
\hline & 87.5 & -2.7 & 86.5 & -2.6 & 86.3 & -2.7 & 80.3 & -4.8 \\
\hline & & 9.0 & & 8.7 & & 9.5 & & 10.3 \\
\hline \multirow[t]{3}{*}{$\mathrm{R} 2 \mathrm{a} \beta 2$} & & -6.0 & & -5.9 & & -6.0 & & -5.9 \\
\hline & 30.1 & -4.9 & 25.6 & -4.3 & 30.2 & -4.7 & 29.9 & -5.3 \\
\hline & & 10.9 & & 10.2 & & 10.7 & & 11.3 \\
\hline \multirow[t]{3}{*}{$\mathrm{R} 2 \mathrm{a} \beta 3$} & & -4.7 & & -5.1 & & -4.9 & & -5.2 \\
\hline & 10.5 & -4.6 & 10.6 & -4.6 & 10.2 & -4.8 & 13.9 & -4.4 \\
\hline & & 9.3 & & 9.7 & & 9.7 & & 9.6 \\
\hline \multirow[t]{3}{*}{$\mathrm{R} 2 \mathrm{~m} \beta 1$} & & -4.7 & & -5.0 & & & & \\
\hline & 131.4 & -3.2 & 124.2 & -2.8 & & & & \\
\hline & & 7.9 & & 7.8 & & & & \\
\hline \multirow[t]{3}{*}{$\mathrm{R} 2 \mathrm{~m} \beta 2$} & & -4.6 & & -4.9 & & & & \\
\hline & 69.2 & -3.6 & 73.2 & -3.1 & & & & \\
\hline & & 8.2 & & 8.0 & & & & \\
\hline \multirow[t]{3}{*}{$\mathrm{R} 2 \mathrm{~m} \beta 3$} & & -4.2 & & -4.1 & & & & \\
\hline & 12.5 & -3.1 & 6.9 & -3.5 & & & & \\
\hline & & 7.3 & & 7.6 & & & & \\
\hline
\end{tabular}


Table 3: Comparison of the calculated and the experimental HF tensors of the alanine radical $\mathrm{R} 2 .{ }^{1} \mathrm{H}_{\beta}$-av. is the the average $\mathrm{HF}$ interaction of the methyl group protons. (See Fig. 1 for the radical structure and Table S2 in the Supplementary Information for the complete tensors.) Principal values of the HF interactions are expressed in $\mathrm{MHz}$ and the deviation angles $\delta$ - in degrees.

\begin{tabular}{|c|c|c|c|c|c|c|c|c|c|}
\hline \multirow{4}{*}{ DFT } & & & \multirow[b]{2}{*}{$\begin{array}{l}\text { Iso } \rightarrow \\
\text { Aniso }\end{array}$} & \multicolumn{3}{|c|}{ Ref. [14] } & \multicolumn{3}{|c|}{ Ref. [9] } \\
\hline & & & & 118 & $\begin{array}{l}6.39 \\
0.70\end{array}$ & -188 & -289 & $\begin{array}{c}70.8 \\
-270\end{array}$ & 5.61 \\
\hline & $\begin{array}{l}\text { lensor } \\
{ }^{14} \mathrm{~N}\end{array}$ & ISO & $\begin{array}{c}\text { Aniso } \\
-1.20\end{array}$ & $\frac{1.10}{5}$ & & & & & \\
\hline & & -3.31 & -0.88 & & 15 & & & & \\
\hline & & & 2.08 & & & 10 & & & \\
\hline & ${ }^{1} \mathrm{H}_{\beta}$-av. & & -2.79 & & & & 6 & & \\
\hline & & 68.10 & $\begin{array}{r}-2.27 \\
5.06\end{array}$ & & & & & 6 & 1 \\
\hline
\end{tabular}




\section{TOC GRAPHIC}

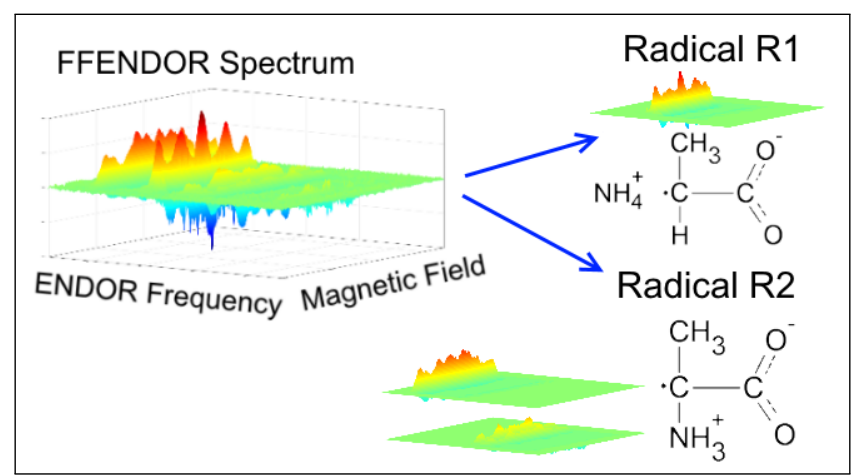

